\title{
Rich afterimage illusion and inflow on-screen mechanisms for visual field constancy and visual orientation in space
}

\author{
Gary M. Zenkin ${ }^{1 *}$ \\ ${ }^{1}$ Institute for Information Transmission Problems, (Kharkevich Institute) of Russian Academy of Sciences, Moscow, Russian Federation
}

\begin{abstract}
It has been shown with using a complex 3-D configuration of reference points, that, in the presence of rich afterimage (rA), eye movements lead to transformation of the visual field, representing a complex rotation and scale transformation of 3-D space with a center somewhere in the region of a hypothetical "cyclopean eye". Experiments have shown that this also results in the recalculation of the coordinates of the observer's gaze direction, entirely based on inflow information, independent on the signals of the oculomotor efferent system. Three types of rA illusions are described under different kinds of eye movements. A model is again (after 1976) expressed that these phenomena reflect the work of the "contextually" independent Screen Constancy Mechanisms (SCM), which operate at the hypothetical Constant Visual Screen (CVS) level. This level can still be considered as "pseudo-sensory" one of the visual system, located before the formation (appearance) of the object-structured visual field. In experiments, certain conditions affecting the degree of afterimage "richness» have been also investigated.
\end{abstract}

\section{Introduction}

The special qualities of a structurally rich positive afterimage (rA), unlike a small (or poor) one, attracted attention relatively recently [1-3] in comparison with the long history of a detail study of visual afterimages [4]. Rich afterimage takes most of portion or full visual field and is practically indistinguishable from a real visual scene illuminated by a gently fading source of bluish color.

As described in our previous papers [1,2] it has been shown in experiments that in the presence of the $\mathrm{rA}$ change of the fixation point in the frontal plane or in depth led to disruption of the real space perception constancy. It has been expressed in the fact that the saccadic eye movements when changing the fixation point did not affect the position or size of rA, but led to the apparent jump of the actually stationary luminous points in the opposite direction and even change their apparent size. This phenomenon has been called "the rich afterimage illusion" [2].

It could be interpreted as a change of the coordinate grid (remapping) of the internal visual space (visual field) and thus real physical space model, which has been confirmed in carried out experiments by the relevant disorders of the subject's motor orientation. It is important to note that in these conditions there have been also recalculation of the observer' gaze direction, which leads to an obvious contradiction between the direction of the gaze and eye position in the orbits. This fact would pointed out that the calculation of the gaze direction can be an independent procedure, does not work-related with signals of oculomotor efferent system.

In the experiments carried out previously the fixation points has been used was actually pretty poor one-dimensional configuration, which is why it was impossible to determine how accurate conversion of the internal three-dimensional space model occurred in this abnormal conditions. It was unclear for example whether the apparent change of the light points position in the frontal plane is reduced to transform the coordinates by parallel shift or this transformation could be represented as the rotation of the entire visual field grid around a vertical axis located somewhere in the observer' head.

In current experiments, more complex 2-D or 3-D configuration of the luminous points ("reference" ones) were used, which the author believe would help to identify in more details the nature of the apparent transformation of the actual visual space on the one hand and on the other hand also apparent recalculation of the observer' gaze direction regardless of the oculomotor system signals. In addition, we investigated the influence of some parameters of the $\mathrm{rA}$ on the exhibited illusions for subjects with different state of the individual binocular system.

\section{Methods}

In the experiments, the afterimages were elicited by a short $(\sim 10$ ms) flash of light from one two or three studio flashtubes Grifon-150 and Grifon-300. The flash illuminated an entire experimental room containing plenty of visible decor, and a rich, highly articulated 3-D afterimage was produced. The afterimage, arising in conditions of deep dark adaptation, underwent two stages. The first one was color very short and uncertain. In the second stage, a plain gray-bluish positive afterimage developed during a relatively long time and then, after reaching maximum clearness, slowly faded out. In conditions of optimal dark adaptation, the duration of a highly visible positive afterimage was around $10-20 \mathrm{~s}$ and in some way depended on the particular features of the subject's binocular vision.

The rich afterimage covered the entire visual field of observer; it was volumetric and made a strong impression on the subjects. Usually nai've (inexperienced) observers were convinced that the whole room

Correspondence to: Gary M Zenkin, Institute for Information Transmission Problems, (Kharkevich Institute) of Russian Academy of Sciences, Moscow, Russian Federation; E-mail: imz@paragon.ru

Key words: afterimage, eye movements, field constancy mechanisms, reference points

Received: May 03, 2017; Accepted: May 25, 2017; Published: May 29, 2017 
was really illuminated by a slowly fading bluish light source. In the experimental room there were some furnishings at different distances from the subject. In front of the subject, at a middle distance of $1.5 \mathrm{~m}$, were placed number (up to 10) LEDs that looked like a low-luminous red point, each of which could be designated as a start or and fixation point according to the instruction given to the subject.

Luminous points were aligned in the frontal plane or in depth with a distance of $30-40 \mathrm{~cm}$ (about $12^{\circ}$ ) between neighboring ones and were arranged into 3 autonomous blocks. Each point could be a point of fixation or be a coordinate test point in the space as a marker, whose behavior could talk about the details of the transformation of the visual field in the presence of rA as the background. Each point could be independently from others switched on and off at different time in relation to the fire of the flashtubes or development of afterimage, allowing to find out the details of the time-course of the inner visual space transformation or a subjective feeling of eye position in time.

The subject was instructed to fix his gaze on one of the luminous points and to produce one or more of the following actions within the specified interval after the flash: (a) shifting gaze from the initial fixation point to another one in frontal plane or in depth, (b) tracking a moving fixation point, (c) closing by some way one eye to watch visual scene monocular, (d) use a semi-matt translucent flap at the time of the flashtube fire to arrange fuzzy blurred afterimage in various degrees and also some others actions.

The subject was asked to focus attention to the visible changes between the luminous points and an afterimage due to these actions as well as to their own feelings about the eye positions in the orbits and the apparent direction of the gaze. The subject's verbal reports on the observed phenomena and the online dialogue with the experimenter were recorded for further analysis. In some cases, portable light point as some space marker was used for measuring procedures. The experimenter or subject himself could move that point manually, placing it in the desired position in the modified visual field.

All together 12 subjects, male and female from 11 to 60 years old took part in tests. They had different state of their binocular system. It extended the range of observed phenomena, the most important of which will be described in this (short) article. It should be noted that some of subjects had to be pretrained and gain experience of stable fixating a light point during movements, in the presence of a rich afterimages as background. Each subject was tested in several series of experiments, each of which refers to a certain type of illusion and four participated almost in all.

\section{Results and primary online discussion}

In the paper we describe only three main trials including a humbler of subseries, in which various aspects of the impact of rA and eye movements on the visual space perception and the experience of the gaze direction feeling, determine the observer's orientation in space.

\section{Experiment 1}

The illusion of "horizontal space rotation" under horizontal change of the fixation point in the frontal plane (Figure 1).

In the figure $2 \mathrm{~b}$ clearly visible turn of the blocks a (1-3) and $\mathrm{b}$ (14) is noteworthy according to their "new position" in the transformed space and appropriate in visual field. Such views of visual field after the change of the fixation point was reproduced under strict control of the observer: the last to confirm the such an illusory picture if one really experienced the illusion. In this experiment subjects noted particularly the fact of turn of each block of points. (a and b) and this feature attracted their attention at the initial moment especially.

Thus, the illusion can be described as a result of the 3-D space rotation about a vertical axis, located somewhere in the head area (cyclopean eye point). Here it is the very time note that this transformation is back to those optical transformations on the eye retinas that occur under real vision conditions. Then each subject reported that the changing the fixation point has not led to a change in the direction of one's gaze: there was an overall impression that one was still looking straight ahead or at the same point in space as before.

Sometimes, it was the reason why some subjects doubted that they really have done to change the point of fixation. Also some of them described the obvious discomfort due to the contradiction between the direction of the gaze and sense of eye position in the orbits.

Finally, it should be especially noted that the illusion has ability to manifest itself significantly longer than the time of the afterimage existence, in fact in total darkness (see the time-cadres in Figure 2).

In this final period of illusion fading, just in the absence of an afterimage, the subjects noted a particularly distinct discomfort in the eye area, sometimes resulting in a short-term headache.

Six modifications of this experiment have been carried out. All of them are described below but some of them only in short manner.

(i) In this trail a subject has not seen any luminous point (Figure 2 B) from the very beginning of the experiment. In the full dark one fixed the eyes in usual position on the base of previous experiences (Figure 2B (a)) and after flash made "change of the fixation point" in familiar manner. Immediately after or sometime with long delay (as it is shown in the Figure 2B (c)), full point configuration reappeared, and a subject could watch them and describe the relative position in the transformed field.

The experiment showed that all parameters of the illusion were the same in this conditions and it again looked like a 3-D-turn of the space. The same was with the sense of contradiction between the gaze direction and eye position in the orbits.

Thus, it can be seen that the mechanisms exhibiting their activity under these conditions do not analyze ("take into account") the state of individual reference points, but deal with the whole 3-D space as geometric point manifold.

(ii) In this modification, the subject had to change the fixation point not with an saccade but by turning the head. In this case, the eyes practically did not change their position in the orbits. From the previous work [1] it was known that head movements substantially change the observed phenomena. Something similar we could wait for here, However the results of this series have exceeded all our expectations. As can be seen in Figure 3 change of the fixation point by the head turn leads to the same illusion of a rich afterimage: the reference points make a jump in the opposite direction and the afterimage remains stationary. At the same time, the subject feels that his head has remained in the previous straight ahead position. This phenomenon persists up to significant angles of head rotation (sometimes up to 60 angular degrees).

In this case, too, the illusion has been retained for some time after complete attenuation of $\mathrm{rA}$. And the subject reports the experience of an apparent virtual keep of the former head orientation with a smooth return it to its actual turned position after sometime. 


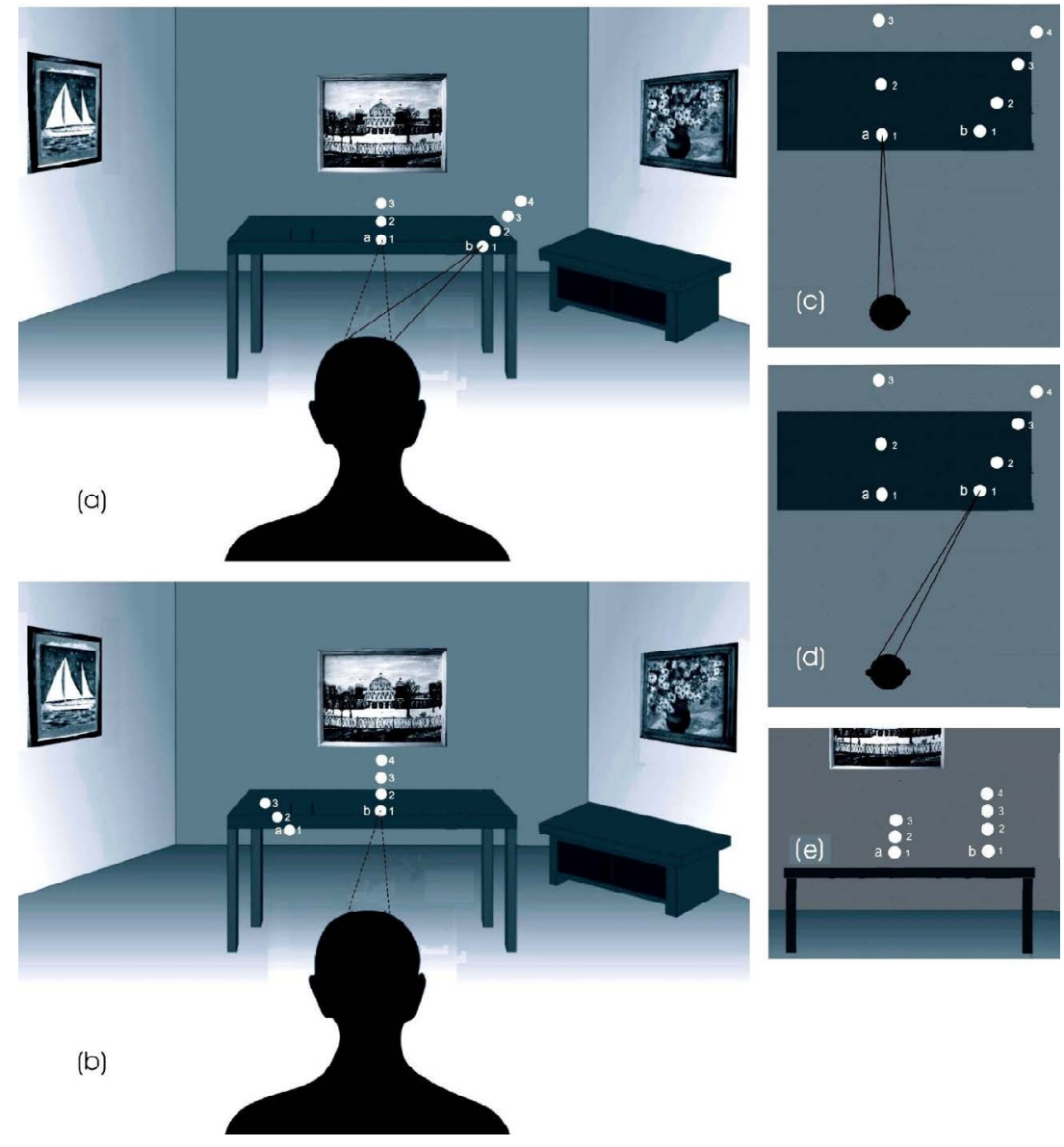

Figure 1. The illusion of "horizontal space rotation" under horizontal change of the fixation point in the frontal plane;(a) view of the subject's visual field at the phase of the optimal development of the rich afterimage; subject to change fixation from point a1 to b1;(b) an apparent shift of the actually stationary light points in the opposite direction with a corresponding change in orientation of the light point blocks; a subject does not notice the real change of his gaze direction;(c), (d) the actual location of the light points and gaze direction in the start (c) and the end (d) phases of the experiment (top view); (e) view of the light point blocks from the subject's position.
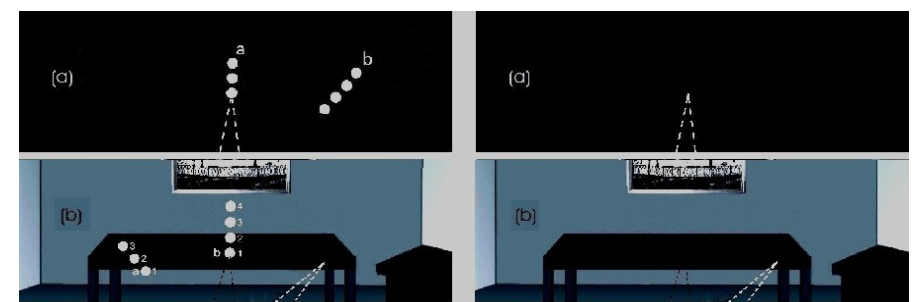

A

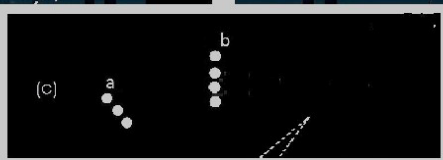

B
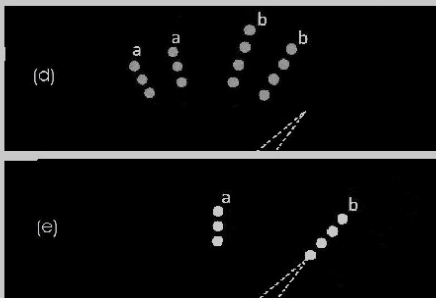

Figure 2. Schematic representation of the time course of illusion when the fixation point is changed horizontally;A- under the permanent presence of light points in the observer's visual field; B- the reference points appear in the visual field after the complete attenuation of the afterimage;(a), (b) the visual field view before flashtube fired and in the moment of optimal development of afterimage (two variants);(c) phase of the illusory but stable position of the reference points, lasting 15 or more seconds;(d) - transient phase of illusion after stable phase (c); in this phase the subject watches a "real" movements of the light points, which lasts no less than 5 seconds; (e)- the final restoration of the real position of the light points in space after at least 20 seconds beyond the complete attenuation of the afterimage; the subject discovers that his eyes are turned to the right. 


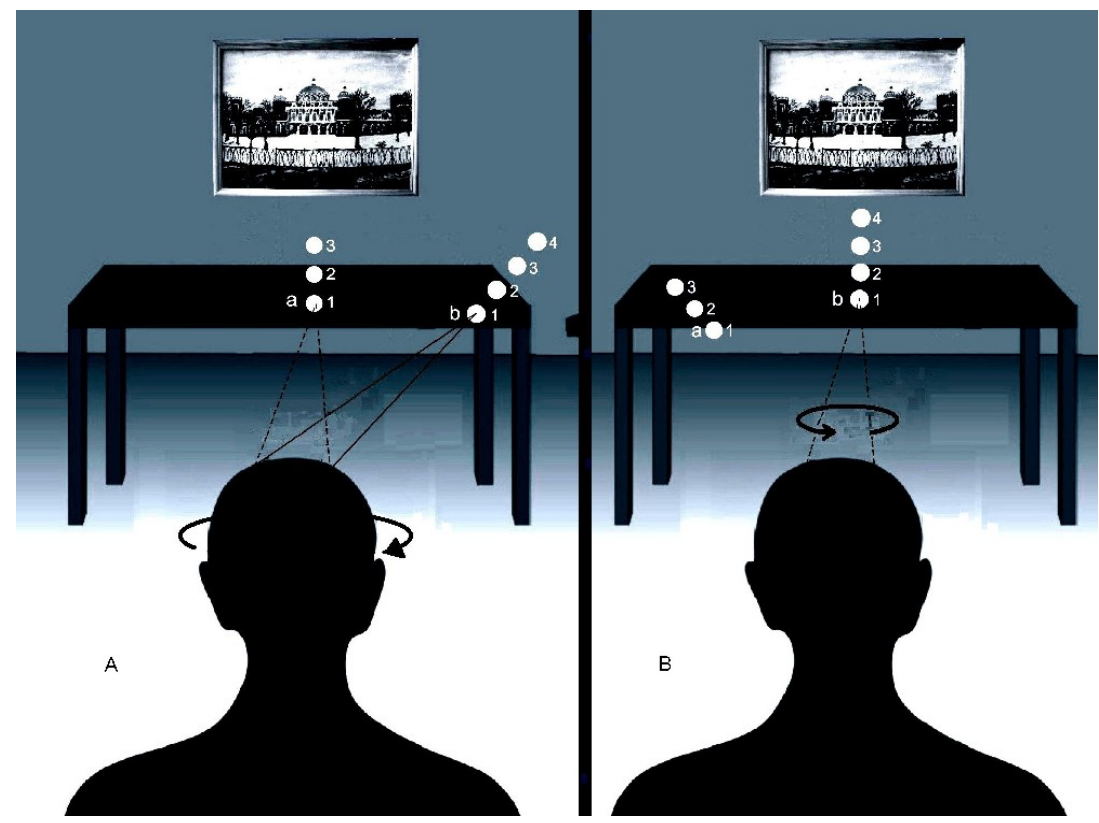

Figure 3. A rich afterimage illusion under the head turn; $\mathrm{A}$ - the subject turns his head after flashtube fired to change fixation point from a1 to b1; $\mathrm{B}$ - the reference points make a jump in the opposite direction, while the head, apparently, maintains its former straight aheadposition.

It should be noted, however, that when the head turns to a point outside the field of the current $\mathrm{rA}$, the latter loses its stationarity and unfolds in space along with the rotation of the head. The same has been seen in [1]. The rotation of the blocks of light points in this test also occurs around the vertical axis, but its position however is likely different from that under the eye saccades. In such experiments there is the potential to determine the exact position of the rotation center, using the features of a specially organized field of a rich afterimage (but it has not performed in current work).

(iii) In this trail, the possibility of manifesting an illusion in the monocular observation of $\mathrm{rA}$ has been tested. One eye of the subject, by his own choice, was closed by the subject himself with the help of a flap or directly with his own palm. Subjects who had an illusion under binocular presentation were constantly informed that they had not seen any apparent difference in the illusion with the monocular variant: monocular illusion was observed stably and also a considerable time remained after the attenuation of the afterimage. However, some subjects reported a loss for them in the illusory scene of its depth: the blocks of the light points looked more likely to be vertically disposed than those went into the depths. Another unexpected feature has been manifested in some subjects with some impairments of binocular vision, which, under normal daylight conditions, did not create any discomfort for them. In experiments with binocular $\mathrm{rA}$, these subjects underwent no illusions or their impressions were vague and difficult to describe. But with monocular observation, two such subjects reported, the illusion appeared and was stably observed.

(iv) In this trail, the influence on the illusion of geometric clarity of rA has been tested, which in our opinion determines what was called structural richness. In this series, only one subject experienced with a reliably expressed illusion took part. The quality of $\mathrm{rA}$ was varied using a semi-matt translucent flap with a different haze value. The flap was located in front of the subject's eyes just before the flash and immediately afterwards was removed. The subject made as usual the change of the fixation point against the background of already fuzzy in varying degrees $\mathrm{rA}$ (It looks like defocused scene as it happens when looking through the misted glasses).
Three degrees of fuzzy have in total, been used by the single-, double- and triple-layer semi-matt flap. In the first case, the subject reported that he quite satisfactorily distinguishes the details with the edges that have lost their sharpness, while in the remaining two, the distinction of parts sharply fell. Correspondingly, for all 4 states of the extended afterimage, the percentage of manifestation of the illusion can be approximately estimated by several tens of submissions for the subject $\mathrm{AB}$ as $100 \% \sim 80 \% \sim 20 \%-0 \%$. Figure 4 shows as only a visual illustration fuzzy extended afterimages, in which illusion occurred in only about $20 \%$ of cases.

(v) In this series, the fixation point was changed at the same time with the tilting the head to the shoulder. Interest in this variant of the experiment was explained by the fact that when the head is tilting to the shoulder, the behavior of $\mathrm{rA}$ coincides with the behavior of a poor one $[1,2]$ : the $\mathrm{rA}$ also inclines toward the tilt of the head. At the same time, it retains a well-defined volumetric view.

Our question was whether the inclined $\mathrm{rA}$ would retain its stationary in its new state and whether it would thereby a "rich afterimage illusion" manifests itself. In the trails, the subject, made after a flash, a change in the fixation point simultaneously with the tilt of the head in the same direction as the saccade or according to the instruction received in the opposite one. As shown in Figure 5A illusion takes place and this occurs regardless of the relative inclination of the head.

In this case, the change of the fixation point horizontally from point a 1 to b1 leads to a more complex saccadic motion, but regardless of that a horizontal jump of the real space and the illusion of keeping the direction of the eye despite changing the position of the eyes in orbits manifested itself completely and also with its prolonged delay after complete attenuation of $\mathrm{rA}$.

(vi) The same question we had with the situation where the change of the fixation point occurs when the observer approaches at the same time the reference points. It is known [1,2] that in this case there is a clear decrease in the apparent size of $\mathrm{rA}$, regardless of its object content, sometimes to very small dimensions. And this completely coincides with the behavior of the poor afterimage. These experiments exactly as 
in the series (v) have shown, that change of the fixation point from al to b1 and simultaneous approach the subject to the reference points does not affect the rA horizontal stability and the illusion manifests itself to the full. An illustration of this fact is shown in Figure 5B.

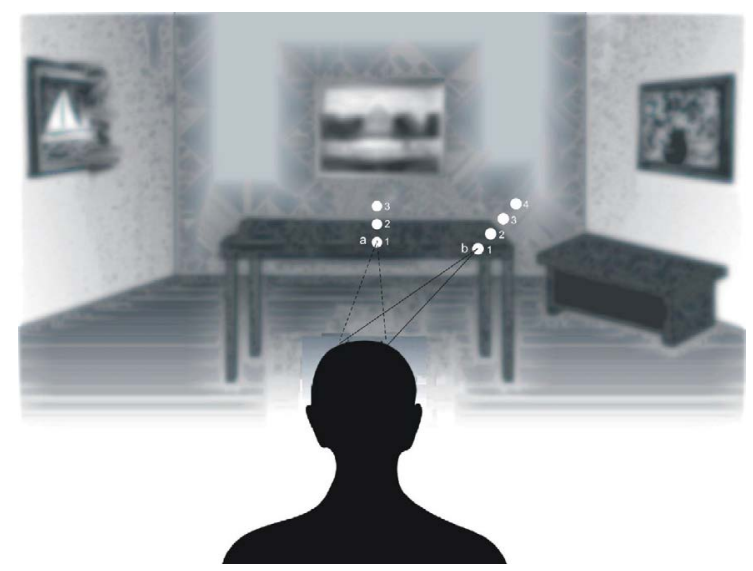

Figure 4. An example of a fuzzy afterimage under which the illusion has not been observed practically; only spontaneously very rarely manifested itself.
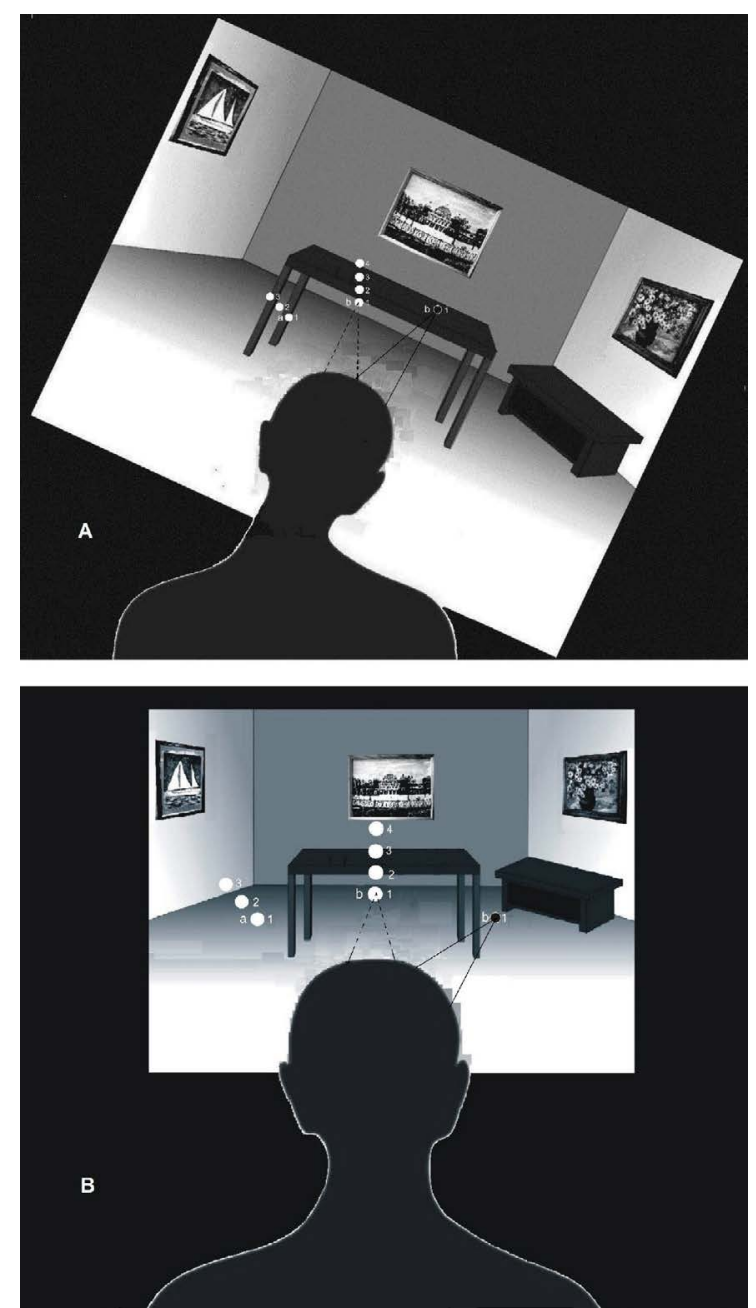

Figure 5. Observed illusions when the fixation point changes from a1 to b1 with movement at the same time of the observer's head; A - when the head tilts to the shoulder; B - when the head approaches the reference points and afterimage is zoomed out.
It should be noted here that figures $5 \mathrm{~A}$ and $5 \mathrm{~B}$ do not accurately reflect the pattern observed by the subject. There are no dark areas in the real situation and the modified $\mathrm{rA}$ occupies the modified visual field of the observer entirely. The dark zones along the edges of the afterimage here are only as illustrative, showing in particular that everything observed occurs in a real darkness.

\section{Experiment 2}

The combined illusion of three-dimensional transformation of space - "space jump" in three directions at once - horizontally, vertically and in depth (Figure 6).

In the experiments of this series, the subject made a change in the fixation points from a1 to b4 and also from b4 to a1. In these cases, the spatial configuration of the light points have been formed in such way (see Figure 6 (c), (d), that after the saccade all three coordinates of the fixation point have been changed essentially for a dozens angular degrees.

In Figure $6 \mathrm{~b}$, in addition to the horizontal and vertical jump of the space a shift of the reference points along the depth is also shown. At the same time, the points appear closer to the observer in zone $\mathrm{A}$ and further away from the observer in zone B. In any of these cases, the subject does not notice the complex movement of his gaze in space and is ready to assert that neither the direction of his gaze nor the depth (range) of the gaze has been changed.

In this case, the apparent size of the points also changes: the size decreases when gaze shifted from the near point al to the far one b4 and increase when the fixation point are reversed from the far point $b 4$ to the near one a1. Everything happens almost as according to the law inverse to the Emmert's law and only in application to real objects but not to their afterimages.

It is important to note here that the change in the coordinate grid of the space along the depth axis leads to an apparent change in the size of the points, which is the artifact of the scale transformation of an entire space, namely, its compression in the variant of experiment $\mathrm{A}$ and its expansion in the variant of experiment $\mathrm{B}$. And the apparent change in the size of points is the result of the work of the size constancy mechanism known in psychophysics. In variant B, some subjects reported that they saw the far point of the block a a3 located behind the frontal wall of the virtual afterimage room, and the point b4 emerging behind the side wall of the virtual room. This again indicates that under conditions of the experiment a large-scale transformation of the entire visual space is taking place.

Thus, we can conclude and argue that a rA, due to its rigid space geometric structure, creates an extremely stable internal virtual space, in relation to which all events that occur in the field are reinterpreted. This space is retained for some time after the expiration of the very afterimage (could remind Figure 2).

\section{Experiment 3}

The illusion of "smooth shift of space" when tracking a moving point (Figure 7). This experiment was usually carried out as the initial stage for most of subjects because it does not require any special action for the subject. This enabled him to get an experience of strict fixation and focusing on the little light points against the bright impressive afterimage, occupying the entire visual field.

Figure 7(b) shows how looks the illusion of motion for most of the subjects: the most of them have seen a moving point as fixed one, while 


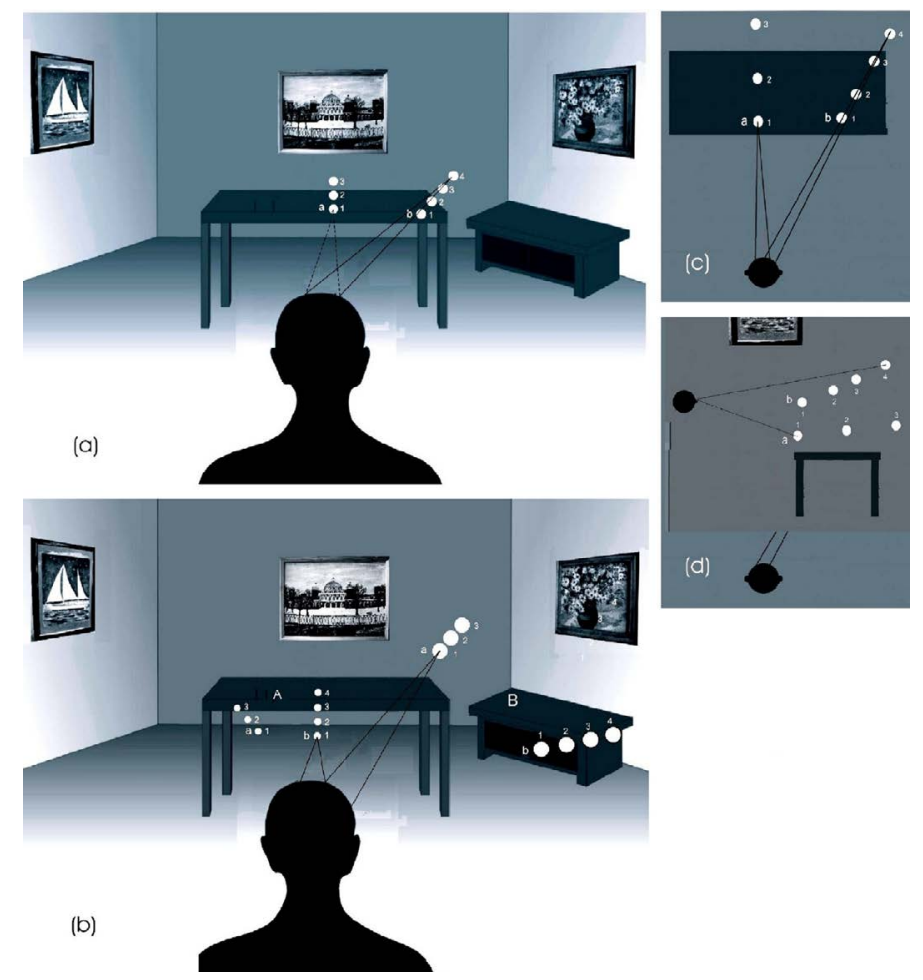

Figure 6. The illusion of a "space jump in three dimensions" when the fixation point is changed in three directions at once; (a) view of the subject's visual field in the phase of optimal development of a rich afterimage; the subject changes the fixation point in one case, from the near-center point a1 to the far side point b4 or in the other case in the opposite way from b4 to a1;(b) an apparent jump and a change in the sizes of actually stationary blocks of the light points in two variants of the experiment: zone A - when changing the fixation point from left to right from bottom to top from near a1 to distant b4; zone B - when looking backwards from right to left from top to bottom and from long distress b4 to near a1;(c), (d) - the actual location of the luminous points and the direction of the subject's gaze in two projections - (c) - the top view and (d) - the side view.

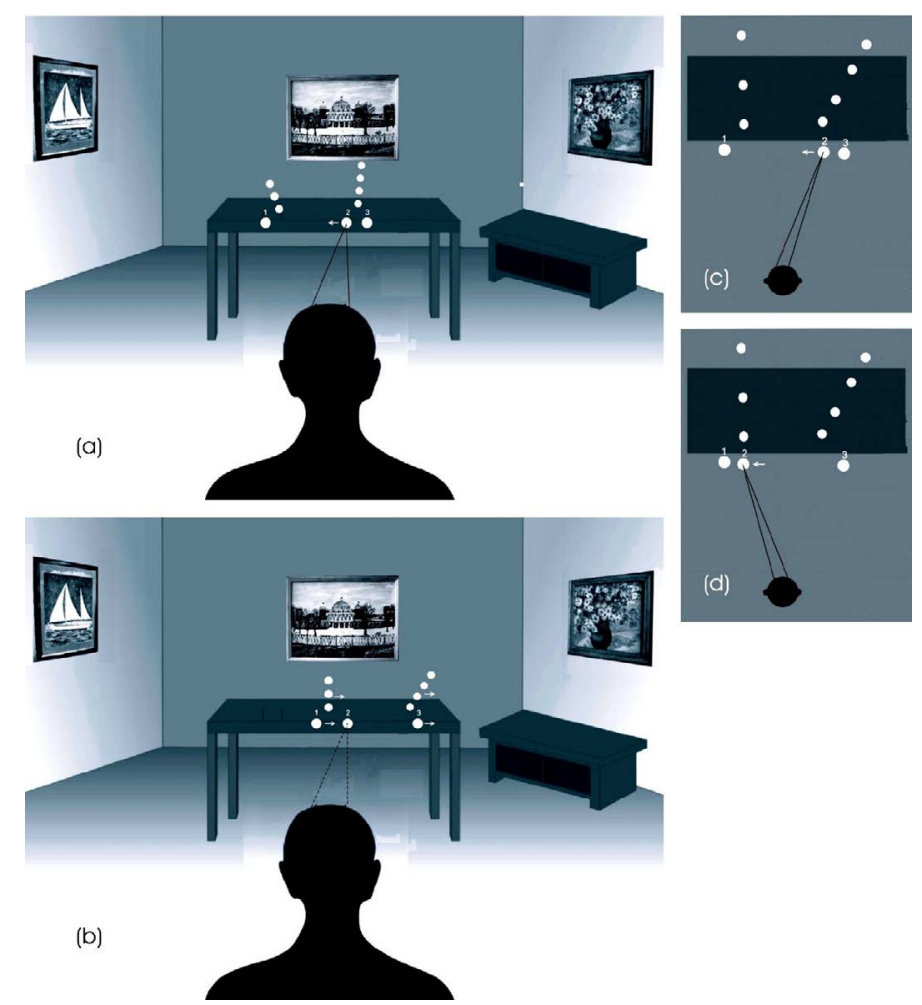

Figure 7. The illusion of the smoothly moving fixation point persuit.(a) The visual field view in phase of the rich afterimage optimal development; a subject tracks smoothly moving point 2 under the rA background;(b) apparent movement of actually stationary configurations of light points in the opposite direction while the moving point 2 looks (like) fixed (stationary); at the same time the subject does not notice the movement of his eyes in their orbits, while feeling that the direction of his gaze has not changed;(c), (d) the actual location of the light points and gaze direction in the start (c) and the end (d) phases of the moving point tracking (top view). 
all other really stationary light points started to move in the opposite direction. It is important to note that each of subjects noticed also an apparent change in the orientation of each block of the alignment points.

\section{It is interesting to describe the dynamics of this illusion.}

When the fixation point began to move immediately after the flashtube fired and before the moment when the rA became good developed the subject saw movement of the fixation point and the stationarity of all others light points. But at a some moment when the rA get of enough quality (richness) the fixation point stops, and the same moment all the rest light points begin to move in the opposite direction. This illusion lasted for a time, almost up to full attenuation of the rA, but then again, the situation is reversed: all light points stayed in their new position (and new block direction) but the fixation point began to move up to stop in its final position. Here we should pay attention to the fact that there is no delay of illusion after the attenuation rA, which has been observed in experiment 1 (see Figure 2). That is, in these conditions, the remapping procedure acts as an active dynamic process.

The entire path of the fixation point movement in the visual field was approximately $30^{\circ}-40^{\circ}$ (about $70 \mathrm{~cm}$ ) and it lasted for a $10-20$ seconds. But above described timing of the illusion it can be changed by adjusting the brightness of the flash or the state of dark adaptation of the subject (and thus the geometric quality of the $\mathrm{rA}$ ).

There was a boundary quality ("richness") of the rA, in which some of the subjects observed an extremely unstable state of the smooth space shift illusion. In this case, the fixation point and the rest ones alternately changed its status (from moving to stop) as if at random moments despite the fact that all this time the fixation point smoothly and continuously moved from one extreme to the other.

It should be noted that, under all its apparent clarity $\mathrm{rA}$ in its detailed drawing of the visual scene markedly inferior to the quality of the visual field in normal daylight vision. Perhaps this relative "weakness" of the rA is a major cause of the illusion instability or even its complete absence in some of the subjects related to the individual characteristics of their visual system. For these subjects the structural power (capacity) of the visual stream realized by $\mathrm{rA}$ inside visual system not enough for the afferent mechanisms of the binocular fusion function for visual field constancy.

\section{General discussion}

So, the phenomenon of rA illusions with all kinds of eye movements indicates that each such movement leads to a recalculation of the coordinate grid of the internal three-dimensional model of the visual space. This conclusion has been drawn by us even in the first experiments [1], but only thanks to the use of a complex composition of reference points in these experiments, it became, in the literal sense of the word, an obvious fact. This obviousness arises from two features of rA.

First, in binocular vision, the structural richness of rA forces the visual mechanisms of fusions to find in the presence of a powerful afterimage the only possible solution for creating a stable and internally consistent visual field in all three dimensions. In the case of monocular vision, the visual memory mechanisms probably perform this function.

Second, the "unexpected" for the visual system, the attachment of rA to the photoreceptor layer forces it to perform a full recalculation of the coordinate grid of the real visual space (remapping). And in this case, the light points act only as markers of real space, as they are still tied to it but not to the photoreceptor field. Immobilized rA forms a new virtual space, false from the observer' point of view and his practical needs, but is only acceptable under these conditions for the mechanisms of the visual system (not only fusional ones) responsible for the visual field stability (constancy).

It is important to note that this also applies to the calculation of the gaze direction, i.e. in fact, the observer himself orientation in the visual and hence physical space. In the conditions of a fixed head eye movements can not affect the global content of the visual scene and they perform the function of transferring the "fovea vision" contribute to the re-design of all new areas of the field with the greatest possible detail. And the inflow mechanisms of constancy under these conditions perform also the function of recalculating the observer's gaze direction in the visual field coordinates, thus providing the "gaze direction constancy ", as well as facilitating help to do this in the coordinates of the body diagram (egocentric coordinates).

This happens as experiments have shown also in the absence of reference points. They act as an auxiliary "fragment" of the experimental situation, as "markers" of that real space, which in fact disappears from the internal representation of the visual system. This is the main important conclusion from the experiments.

How real is the reorganization of the coordinate grid of the space internal model the results impressive show with a long delay of the illusion after the complete attenuation of rA itself, which in fact causes not the "illusion of remapping", but a real reorganization of the internal perceptual model of the visual world. And again the reality of this restructuring is particularly evident for the observer mind during the period of a smooth return to the real space (see Fig. 2 (d)), which lasts sometimes for a many seconds. It is unlikely that this process, which clearly demonstrates the work of the visual field constancy mechanisms, can be attributed to the level of Helmholtz's 'unconscious inferences' [4].

Most likely this is purely perceptual, and maybe even "pseudosensory" level, because here nothing depends on the subject matter (object content) of the field. It means that it is before the appearance of objects in mind, that is, before the subject structuring of the visual field. That is, in other words, this means that this is the level of the almost iconic representation of the visual world as a "point manifold", where the "point" can be a small local geometric feature detected in the point field of photoreceptors.

While 'unconscious inferences' can hardly be attributed to the world that is not yet filled with objects. In particular, illusions with an oblique or reduced in size $\mathrm{rA}$ indicate that as if the visual system still "does not know" about the existence of objects in the world. It "knows" only the geometry of three-dimensional space. On this basis, our hypothesis [1] arose about the existence of a Constant Visual Screen (CVS), which is served by on-screen (pseudo-iconic) mechanisms of invariance (SCM). And this level is responsible primarily for the visual field constancy and for the "gaze direction constancy" (visual direction constancy).

Influence of the parameters of the eye movements and quality of an afterimage (if it poor or rich) on the apparent position and size of both the reference points and the afterimage itself make someone look more closely at the results of experiments with the presence of the body parts (for example, the hand) in the observer's visual field [5]. If the hand in such an experiment appears as an independent small and thus a poor afterimage or as a fragment of a rich one, this can lead to 
completely different visual phenomena. And the quality of the richness or poorness of the afterimage, as we have seen here, depends on many parameters both external (for example, the structural quality of the afterimage field, weak brightness, unclear boundaries) and internal (for example, loss of stable fixation of the chosen point especially when the convergent eye movements and the tension of the accommodation are close to the limiting states)

It means that more precise qualitative control over these parameters is required for valid conclusions about the effect of proprioceptive signals on visible changes of the body part size of an observer. By the way, no proprioceptive signals about the actual position of the turned head could overcome the signals about the state of the virtual visual world under the experimental conditions (see Figure 3).

In the final part of the discussion should be specially emphasized that for all evidence of the phenomena observed in the experiments it takes thorough efforts to draw definitive conclusions about the nature of their origin, of their functional organization and interpretation. This will require further thorough researches. Here we completely avoided the contribution that mechanisms make on the basis of the regular organization of the already visual field of objects $[6,7]$ and the extreme reverse case of the influence of the oculomotor system signals in the ultimately reduced visual environment(a few light points in full dark). Also, the manifestations of the influence of these signals under the drug block of the motor muscles of the eye and thus impairment of the operation of the oculomotor system [8-10]. Interpretation of the results of these studies will require in our opinion an additional analysis in the light of the illusions described here.

\section{Conclusion}

The paper describes three illusions that arise in the observer's visual field when moving the eye in the presence of a extended rich afterimage.

The illusion of a horizontal turn of the 3-D visual space is observed under horizontal change of the fixation point in the frontal plane. The center of rotation is located in the point of the "cyclopean eye".

Illusion persists:

- in the presence or absence of reference light points in the visual field;

- when changing the fixation point by the head turning;

- with binocular or monocular vision;

- with a simultaneous change of the fixation point with the head tilt to the shoulder or the observer's approach to the reference points.

Illusion has the property of remaining long after complete attenuation of the afterimage. The illusion disappears with complete loss of the geometric clarity of boundaries in the afterimage. The complex illusion of a three-dimensional transformation of the visual space, including also its scale transformations arises with a change in all three spatial coordinates of the fixation points.

The illusion of a smooth turn of the visual 3-D space is observed under tracking the fixing point moving in the frontal plane. The coordinates of the observer's gaze direction are recalculated in all kinds of observed illusions, giving him an adequate orientation in the modified virtual space, but completely not corresponding to the actual eye position in the orbit. It is hypothesized that all observed phenomena reflect the activity of screen pseudo-iconic mechanisms of the of the visual field constancy and the observer orientation in the visual space.

\section{Competing interests}

The author declares that he have no competing interests.

\section{Acknowledgments}

The author is grateful to Dr G. Rozhkova for organizational support as well A. Belokopytov especially for comprehensive technical assistance.

\section{Funding}

The author(s) received no financial support for the research, authorship, and/or publication of this article.

\section{References}

1. Zenkin GM, Petrov AP (1976) Transformation of the afterimage by observer movements, constancy of the visual field, and field constancy mechanisms. Human Physiol 2: 925-931. [Crossref]

2. Zenkin GM, Petrov AP (2015) Transformation of the visual afterimage under subject's eye and body movements and the visual field constancy mechanisms. Perception 44: 973-985. [Crossref]

3. Pelz JB, Flayhoe MM (1995) The role of exocentric reference frames in the perception of visual direction. Vision Res 35: 2267-2275. [Crossref]

4. Balonov L Ya (1971) Afterimages. Leningrad, Russia: Nauka Press (in Russian).

5. Boring E (1942) Sensation and Perception in the History of Experimental Psychology. N.-Y.: Appleton-Century-Crofts.

6. Bross M (2000) Emmert's law in the dark: active and passive proprioceptive effects on positive visual afterimages. Perception 29: 1385-1391. [Crossref]

7. Allport AP (1995) Theories of perception and the concept of structure. N.-Y.: Wiley $709 \mathrm{pp}$.

8. Gibson JJ (1979) The ecological approach to visual perception. Boston: Houghton Mifflin. $332 \mathrm{pp}$.

9. Stevens JK, Emerson RC, Gerstein GL, Kallos T, Neufeld GR, et al. (1976) Paralysis of the awake human: visual perceptions. Vision Res 16: 93-98. [Crossref]

10. Matin L, Picoult E, Stevens JK, Edwards MW, Young D, et al. (1982) Oculoparalytic Illusion: Visual-Field Dependant Spatial Mislocalizations by Humans Partially Paralyzed with Curare. Science 216: 198-201. [Crossref]

Copyright: (C2017 Zenkin GM. This is an open-access article distributed under the terms of the Creative Commons Attribution License, which permits unrestricted use, distribution, and reproduction in any medium, provided the original author and source are credited. 